\title{
Wear law in mixed lubrication based on stress-promoted thermal activation
}

\author{
Xin PEI ${ }^{1}$, Wei PU ${ }^{1, *}$, Jialong YANG ${ }^{1}$, Ying ZHANG ${ }^{2}$ \\ ${ }^{1}$ School of Aeronautics and Astronautics, Sichuan University, Chengdu 610065, China \\ ${ }^{2}$ School of Manufacturing Science and Engineering, Sichuan University, Chengdu 610065, China \\ Received: 15 October 2019 / Revised: 12 December 2019 / Accepted: 16 January 2020 \\ (C) The author(s) 2020 .
}

\begin{abstract}
Although several empirical wear formulas have been proposed, theoretical approaches for predicting surface topography evolution during sliding wear are limited. In this study, we propose a novel wear-prediction method, wherein the energy-based Arrhenius equation is combined with a mixed elastohydrodynamic lubrication (EHL) model to predict the point-contact wear process in mixed lubrication. The surface flash temperature and contact pressure are considered in the wear model. Simulation results are compared with the experimental results to verify the theory. The surface topography evolutions are observed during the wear process. The influences of load and speed on wear are investigated. The simulation results based on the Arrhenius equation relationship shows good agreement with the results of experiments as well as the Archard wear formula. However, the Arrhenius equation is more accurate than the Archard wear theory in some aspects, such as under high-temperature conditions. The results indicate that combining the wear formulas with the mixed EHL simulation models is an effective method to study the wear behavior over time.
\end{abstract}

Keywords: mixed lubrication; sliding wear; Archard; Arrhenius; surface topography

\section{Introduction}

Sliding wear is one of the main causes of mechanical component failure. The wear process is caused by a variety of complex physical and chemical changes on the surfaces of contact components and it typically changes with the moving distance. In engineering, contact pairs are usually lubricated. The elastic hydrodynamic effect of the lubricant separates the contact pairs; however, partial asperity contacts remain owing to the rough peaks of the surfaces, which may lead to wear. Therefore, there is a need to investigate the wear in mixed lubrication.

Although the mechanism of wear has been studied for three centuries and numerous empirical formals have emerged, the understanding of wear is still limited [1]. Among the empirical formals, the Archard wear law [2] is the most accurate for predicting adhesive sliding wear. According to the Archard theory, wear has a simple linear relation to load, relative sliding distance, and the hardness of the material. Combining the Archard theory with contact mechanic formulas has been proven to effectively predict wear [3]. Although this theory is consistent with the wear results in engineering, it cannot explain how the material is removed, and it is independent of temperature. With the advent of atomic force microscopy [4] in the 1980s, the wear behavior can be evaluated at the nanoscale. Gotsmann and Lantz [5] showed that the Archard wear law may not be the best method to explain single asperity wear behavior, and they believed that wear is related to energy

* Corresponding author: Wei PU, E-mail: pwei@scu.edu.cn 
barriers and contact shear stress. Their model, based on the Arrhenius equation [6], has been validated by numerous experiments [7-9]. Valentin and Roman [10] also found that the dependencies of the wear volume on the normal force are power-law dependencies which deviate from Archard law. The wear mechanism explained by the Arrhenius relationship is an important advancement for understanding atom-by-atom wear. Jean-François et al. [11] have revealed the transition from ductile shearing of an asperity to the formation of a debris particle by molecular simulation. However, most current studies have focused on the study of dry contact or boundary wear $[12,13]$, and few studies have considered asperity contact in the mixed lubrication state, which is common in engineering.

Wear changes the topological structure of the surfaces in real-time. The changes in the surfaces affect the mixed lubrication state and asperity contact, which can immediately affect the wear process. Thus, it is difficult to describe this process with a time-independent model. With the development of mixed elastohydrodynamic lubrication (EHL) models (such as those proposed by $\mathrm{Hu}$ and $\mathrm{Zhu}$ [14], Hooke et al. [15], and Masjedi and Khonsari [16]), the distribution of contact pressure, elastic deformation, and temperature can be predicted based on scanned engineering surfaces as a function of time or frequency. $\mathrm{Hu}$ and Zhu [14] represented a significant advancement in predicting the dynamic lubrication state. In their studies, three-dimensional (3D) asperity surfaces are used, and the contact zone is divided into the EHL and asperity contact regions. The influence coefficient method, which can be accelerated with fast Fourier transform (FFT), is used to calculate the elastic deformation and pressure. In the EHL region, the Reynolds equation is applied. In the asperity contact region, where the gap is 0 , the EHL pressure is automatically reduced to 0 , and the contact pressure is solved using the EHL pressure as the boundary condition. Zhu et al. [17] and Pei et al. [18] combined the Archard theory with the deterministic mixed lubrication model [14]. Zhao et al. [19] found that temperature plays an important role in the wear behaviors of $\mathrm{Cu}$-based friction pairs by an oil spectrum analysis. Morales-Espejel et al. [20] and Lewis [21] considered sliding wear and figure wear in a contact failure simulation. Bazrafshan et al. [22] simulated the wear of multiple rough peaks using a defined adhesion coefficient based on Ref. [23]. Akchurin and Bosman [24] developed a deterministic model to simulate the growth and wear of tribofilm. Furustig et al. [25] developed a two-scale wearing-in model to study the running-in behavior of hydraulic motors in mixed lubrication. Their theory was experimentally verified.

This study aims to evaluate the wear process in mixed lubrication caused by asperity contact. Stresspromoted thermal activation is employed to determine the material loss in the asperity contact area. The simulation results obtained from the combination model of the mixed EHL and Arrhenius equation are verified through experiments and compared with Zhu's Archard-based wear model [17]. In this study, the evolution of the wear profile and wear volume over time is studied. The wear under different speeds and loads at the same sliding distance is compared. Some assumptions are made to simplify the model: 1) only asperity contact causes wear; 2) the wear rate corresponds to the Arrhenius equation; 3) the wear debris quickly flows out with the oil and does not affect the subsequent wear process (ignoring three-body wear); and 4) the materials are isotropic and uniformly distributed.

\section{Mixed EHL wear model}

\subsection{Basic equations}

One of the key steps of the present model is to solve the Reynolds equation. To simultaneously solve the pressure and gap distribution of the EHL and asperity contact regions, a general form of the Reynolds equation proposed by $\mathrm{Hu}$ and $\mathrm{Zhu}$ [14] is adopted as follows:

$$
\frac{\partial\left(\frac{\rho}{12 \eta} h^{3} \frac{\partial p}{\partial x}\right)}{\partial x}+\frac{\partial\left(\frac{\rho}{12 \eta} h^{3} \frac{\partial p}{\partial y}\right)}{\partial y}=U \frac{\partial(\rho h)}{\partial x}+\frac{\partial(\rho h)}{\partial t}
$$

In the asperity contact region, the pressure-flow items on the left side of Eq. (1) will tend to 0, and Eq. (1) is reduced to Eq. (2) to solve the asperity contact pressure. 


$$
U \frac{\partial(\rho h)}{\partial x}+\frac{\partial(\rho h)}{\partial t}=0
$$

where $p, h, \rho$, and $\eta$ are the pressure, gap (film thickness), density, and viscosity at each discrete node; $t$ is time. The relationship between the lubricant density and pressure is based on Eq. (3), where $\rho_{0}$ is the lubricant density at atmospheric pressure. The Roelands formula given in Eq. (4) is used to solve the viscosity.

$$
\rho=\rho_{0}\left(1+\frac{0.6 \times 10^{-9} p}{1+1.7 \times 10^{-9} p}\right)
$$

$$
\begin{aligned}
& \eta=\eta_{0} \\
& \times \exp \left\{\left(\ln \eta_{0}+9.67\right)\left[-1+\left(1+p / p_{0}\right)^{0.68}\left(\frac{T_{\mathrm{c}}-138}{T_{0}-138}\right)^{-1.1}\right]\right\}
\end{aligned}
$$

where $\eta_{0}$ and $p_{0}$ are the viscosity at room temperature and pressure, respectively; $T_{0}$ and $T_{\mathrm{c}}$ are room temperature and simulation temperature, respectively. Studying the effect of ambient temperature on the wear reveals that the viscosity changes with the ambient temperature; the isothermal model is used when solving the Reynolds equation.

$h$ is calculated by Eq. (5):

$$
\begin{aligned}
h= & h_{0}(t)+B_{x} x^{2}+B_{y} y^{2}+\delta_{1}(x, y, t)+\delta_{2}(x, y, t)+V(x, y, t) \\
& +W_{1}(x, y, t)+W_{2}(x, y, t)
\end{aligned}
$$

The gap is composed of integrated geometry in the $x$ - and $y$-directions ( $B_{x}$ and $B_{y}$ ), the height of the two rough surfaces $\left(\delta_{1}\right.$ and $\left.\delta_{2}\right)$, elastic deformation $(V)$, and removal height of the two surface materials ( $W_{1}$ and $W_{2}$ ). $h_{0}$ is the normal progressive height that changes with time. The deformation can be calculated by Eq. (6):

$$
V(x, y, t)=\frac{2}{\pi E^{\prime}} \iint_{\Omega} \frac{p(\xi, \zeta)}{\sqrt{(x-\xi)^{2}+(\mathrm{y}-\zeta)^{2}}} \mathrm{~d} \xi \mathrm{d} \zeta
$$

where $\xi$ and $\zeta$ are arbitrary coordinates of the solution area.

The available mixed EHL model can predict asperity contact pressure and contact gap. However, the correct material removal law should be confirmed before simulating the removal of surface asperity.
In recent years, experiments have shown that the contact pressure and atomic reaction rate have an exponential relationship instead of a linear one. Thus, the wear model based on Arrhenius was proposed [26], as shown in Eq. (7):

$$
\frac{\mathrm{d} W}{\mathrm{~d} t}=b f_{\mathrm{a}} \mathrm{e}^{-\frac{E_{\mathrm{a}}}{k_{\mathrm{B}} T}}
$$

where $\mathrm{d} W / \mathrm{d} t$ is the decreasing rate of the contact point, $b$ is the lattice parameter, $f_{\mathrm{a}}$ is the attempt frequency, $E_{\mathrm{a}}$ is the activation energy, $k_{\mathrm{B}}$ is the Boltzmann's constant, and $T$ is the Kelvin temperature.

Based on Eq. (7), the removal height can be simplified by Eq. (8), where $\delta$ is the acceleration coefficient. The total simulation time is short compared to the time taken by the actual wear process; therefore, the wear equation can be defined as

$$
\frac{\mathrm{d} W}{\mathrm{~d} t}=\delta \cdot \mathrm{e}^{-\frac{E_{\mathrm{a}}}{k_{\mathrm{B}} T}}
$$

In the Reynolds equation solution for each cycle, the basis of convergence is given in Eq. (9). The Convergence pressure is also determined as

$$
\frac{\sum\left|P^{\text {new }}-P^{\text {old }}\right|}{\sum P^{\text {old }}} \leqslant 0.1^{4}
$$

The superscript 'old' and 'new' represent the previous interaction pressure and the current pressure at the same time-step, respectively. The balance of load is determined as follows:

$$
\frac{\left|\iint_{\Omega} p \mathrm{~d} x \mathrm{~d} y-w\right|}{w} \leqslant 0.1^{3}
$$

where $w$ is the total load.

Because the friction (or shear) and flash temperature interact with each other, they should be solved simultaneously; however, this solving process is complicated. In the asperity contact region, the friction coefficient is set to a constant, typically between 0.08 and 0.15 . In the EHL region, the Bair-Winer model [27] is used to calculate the lubrication pressure. $\dot{\gamma}$ is shear rate. $\tau$ and $\dot{\tau}$ are the shear stress and the derivative of shear stress with time, respectively.

$$
\dot{\gamma}=\frac{\dot{\tau}}{G_{\infty}}-\frac{\tau_{\mathrm{L}}}{\eta} \ln \left(1-\frac{\tau}{\tau_{\mathrm{L}}}\right)
$$


where $G_{\infty}$ is the limiting shear elastic modulus and $\tau_{\mathrm{L}}$ is the limiting shear stress. Both are functions of pressure and temperature $(T)$ and can be calculated using Dyson's experience formulas [28] as follows:

$$
\begin{aligned}
& G_{\infty}(p, T)=\frac{1.2 p}{2.52+0.024 T}-10^{8} \\
& \tau_{\mathrm{L}}(p, T)=0.25 G_{\infty}
\end{aligned}
$$

A point heat source integration method [29] is used to calculate the flash temperature, and detailed information can be found in Ref. [30].

$$
\left\{\begin{aligned}
T_{1}(\xi)= & T_{\mathrm{b} 1}+\left(\frac{1}{\pi \rho_{\mathrm{A}} C_{\mathrm{A}} u_{1} k_{\mathrm{A}}}\right)^{0.5} \\
& \times \int_{-x}^{\xi}\left\{\frac{k_{f}}{h}\left[T_{2}(\lambda)-T_{1}(\lambda)\right]+\frac{q(\lambda)}{2}\right\} \times \frac{\mathrm{d} \lambda}{(\xi-\lambda)^{0.5}} \\
T_{2}(\xi)= & T_{\mathrm{b} 2}+\left(\frac{1}{\pi \rho_{\mathrm{B}} C_{\mathrm{B}} u_{2} k_{\mathrm{B}}}\right)^{0.5} \\
& \times \int_{-x}^{\xi}\left\{\frac{k_{f}}{h}\left[T_{1}(\lambda)-T_{2}(\lambda)\right]+\frac{q(\lambda)}{2}\right\} \times \frac{\mathrm{d} \lambda}{(\xi-\lambda)^{0.5}}
\end{aligned}\right.
$$

where $q$ is the heat generated by the lubricant shear effect or asperity contact effect. $T_{\mathrm{b} 1}$ and $T_{\mathrm{b} 2}$ are volume temperature of the two contacts, respectively, and $k_{f}$ is the thermal conductivity. $T_{1}$ and $T_{2}$ are surface temperature of the two contacts, respectively. $\rho, C$, and $k$ are density, specific heat, and thermal conductivity. Subscript A and B represent the two contact bodies. $\lambda$ is the one-dimensional conduction coordinate.
If the speed of one surface is $0.0 \mathrm{~m} / \mathrm{s}$, it is changed to $5 \%$ of the speed of another surface when solving the flash temperature to avoid the large error caused by the surface and stationary heat source, and this change is only used for the friction-flash temperature process.

\subsection{Modeling methodology}

In our model, wear damage is calculated from asperity contact, where the gap is 0 , and it is affected by the interface temperature, contact pressure, and relative sliding distance. The interface temperature is the sum of the flash temperature and body temperature. The relative sliding distance is related to the timestep of simulation (time increment and cycles).

As shown in Fig. 1, the distributions of the contact gap, flash temperature, and asperity contact pressure are obtained according to the input working conditions before the introduction of wear. Then, the material is removed according to the Arrhenius relationship. The surfaces in the simulation move with the time-step. The moving distance of the two surfaces is related to the dimensionless time (DT) increment, and the surfaces are refreshed according to the material removal in each cycle. To achieve real-time movement and refresh the surfaces, a three-layer independent grid is introduced to the model, as shown in Fig. 2. The three meshes are the ball surface, disk surface, and solving mesh of the Reynolds equation. The surface meshes are denser and wider than those of the Reynolds equation. Conversion

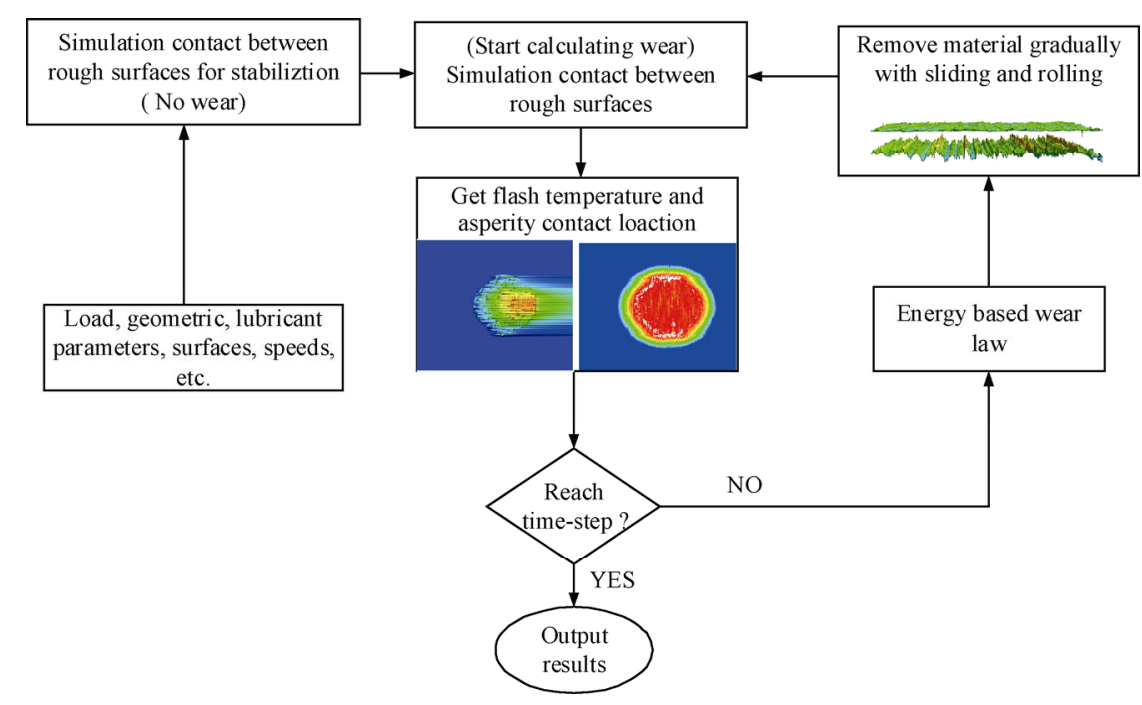

Fig. 1 Flow chart of the dynamic wear model. 


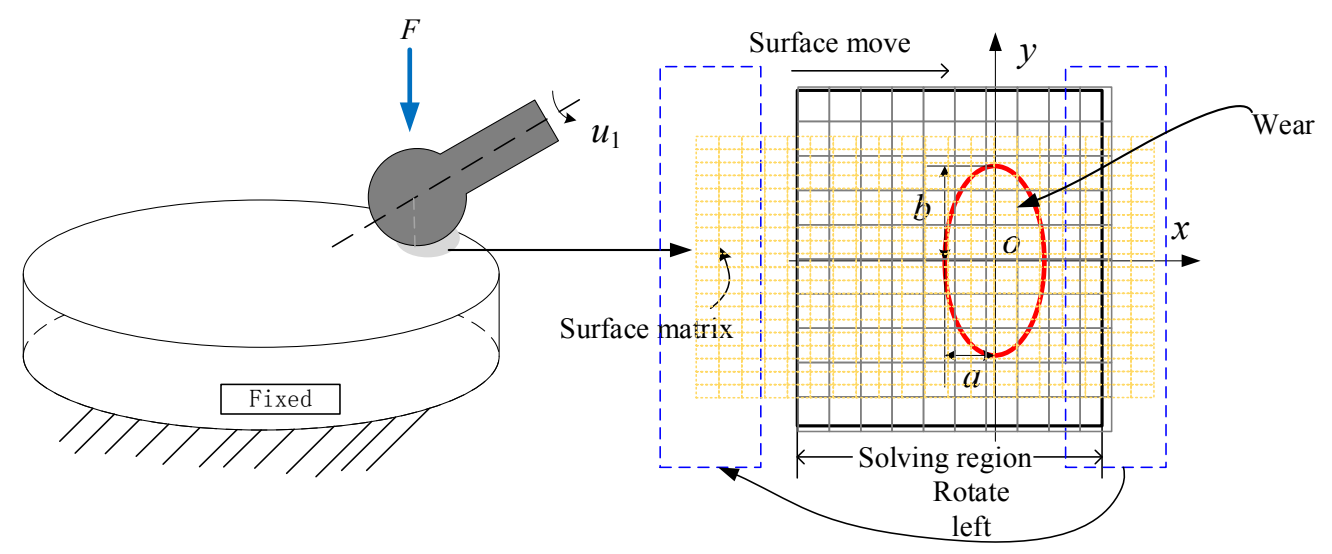

Fig. 2 Three-layer independent grid of the contact area.

of the three meshes is performed using interpolation and mapping [18]. When solving the Reynolds equation, the finite difference method is used, and the two surfaces are interpolated to the solving mesh. Then, the wear is calculated and mapped to change the height of the surfaces at the two surface meshes. The surface moving distance of the ball is fixed at $1.8 \times 10^{-6} \mathrm{~m}\left(\mathrm{DT}=1.8 \times 10^{-6} \mathrm{~m} / \mathrm{a}\right)$ in the present simulation to guarantee the same distance per circle, regardless of the contact width.

The solution domain of the Reynolds equation is discretized into $256 \times 256$ grids. In this study, the region is $\left(-2.2 a_{0}\right)-1.8 a_{0}$ in the $x$-direction (sliding direction) and $\left(-2.0 b_{0}\right)-2.0 b_{0}$ in the $y$-direction (speed vertical direction); $a_{0}$ and $b_{0}$ are the half-Hertz contact width in the $x$ - and $y$-directions, respectively.

\section{Experimental}

The point-wear tests are conducted through balldisk experiments under pure sliding conditions. Oil bath lubrication is used to ensure adequate lubrication, as shown in Fig. 3. This type of lubrication has several

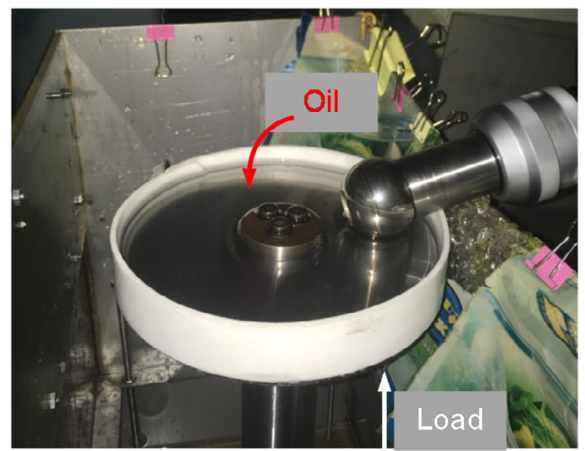

Fig. 3 Experimental device. advantages. For example, the debris is easily discharged with the rotation of the ball, oil shortage is avoided, oxidative wear is reduced, and the working environment is more stable than the injection lubrication.

The wear profiles are scanned using an optical microscope. The wear track on the ball is inconspicuous, almost impossible to observe. This is because of the high hardness of the ball, and the limited wear is evenly distributed in the long tracks. Therefore, only the wear profiles on the disk are considered in the experiments. The total wear volumes are calculated from the central cross-sectional area. The diameter of the ball is $38 \mathrm{~mm}$, the Young's modulus of the ball and disk is $206 \mathrm{GPa}$, and the Poisson's ratio is 0.3 . The hardness of the ball and disk is 4.5 and $2.5 \mathrm{GPa}$, respectively. The root mean square (RMS) roughness $(R a)$ of the ball and disk is 0.05 and $0.3 \mu \mathrm{m}$, respectively. The experiments use base oil without additives. The viscosity of the lubricant is $0.023 \mathrm{~Pa} \cdot \mathrm{s}$ and the density is $0.88 \mathrm{~g} / \mathrm{cm}^{3}$. All experiments are conducted at $T_{0}=27{ }^{\circ} \mathrm{C}$.

\section{Results and analysis}

\subsection{Comparison of wear profiles at different time}

First, the morphologies of the steel disk surface after different wear times are measured. The disk is fixed, the speed of the ball is $0.2 \mathrm{~m} / \mathrm{s}$, and the load is $400 \mathrm{~N}$. Maintaining the same contact location after the measurement is difficult because the measurement requires disassembly of the test piece. Therefore, four sets of experiments are performed 
with wear time of $2,4,8$, and $16 \mathrm{~min}$, respectively, at multiple locations, as shown in Fig. 4.

The wear volume can be calculated from the worn profile, and the wear volume after $4 \mathrm{~min}$ is used to adjust the acceleration factor in the simulation. The adjustment process is described by Zhu et al. [17]. The acceleration factor for the disk is 0.028125 based on the Arrhenius equation. 90 wear cycles in the simulation correspond to $1 \mathrm{~min}$ in the experiment.

As shown in Fig. 5, the wear volume predicted by the Arrhenius relationship is consistent with the experimental results. The wear volume is almost linear with time, corresponding to the stable wear phase. In the early stages of wear (running-in wear), the wear rate is high, and large rough peaks are worn away quickly. Thereafter, the wear rate becomes constant.

Center sections of the wear profile are shown in Fig. 6 to further evaluate the similarities and differences between the experiments and simulations. For the case of sufficient oil supply, the profile of point wear is different from the arc-shaped pit formed under dry contact. For the cross-section of the $y$-direction (assuming the direction of speed is $x$ ), both sides of the wear track are deeper than
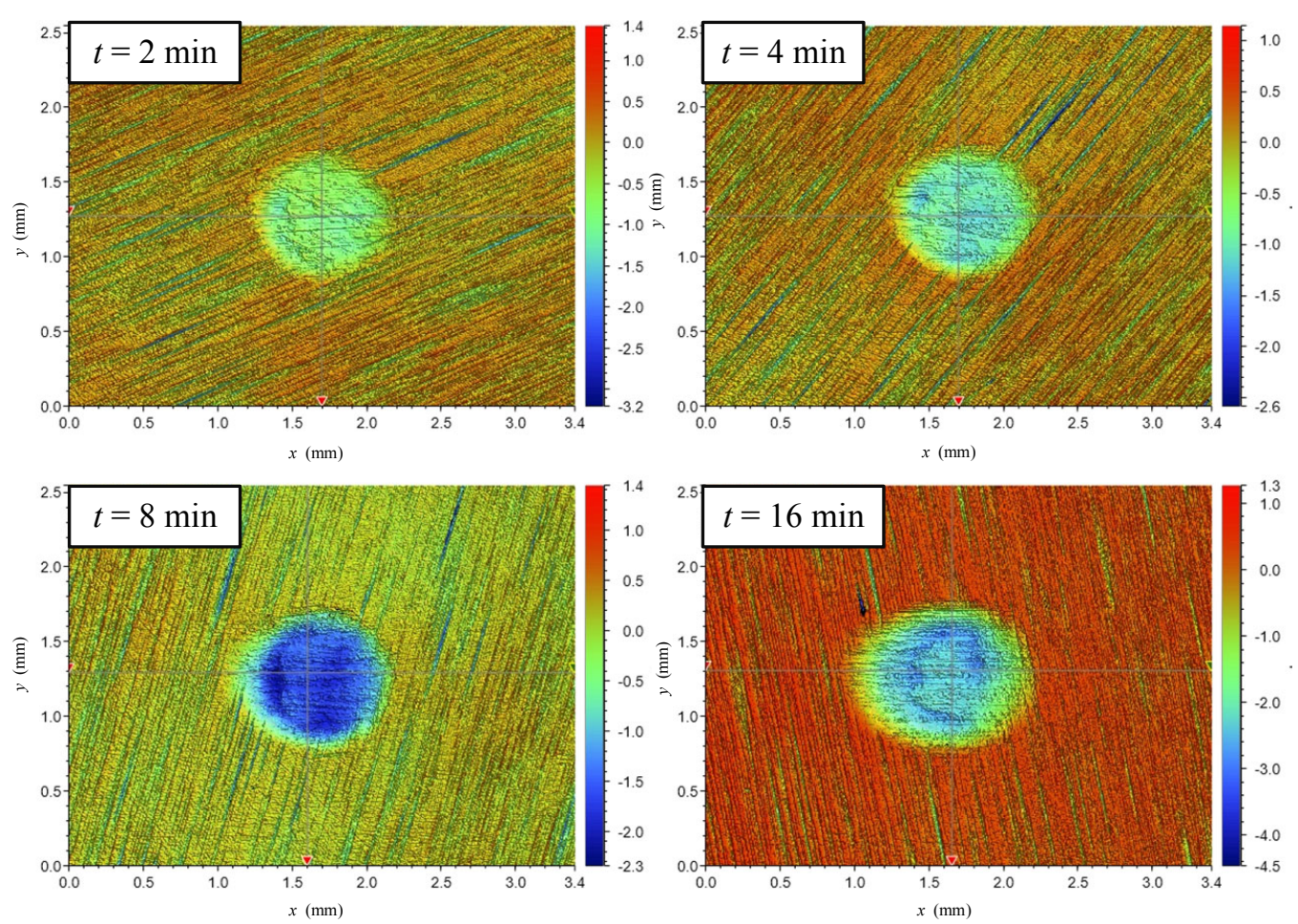

Fig. 4 Scanned disk surface at different wear time.

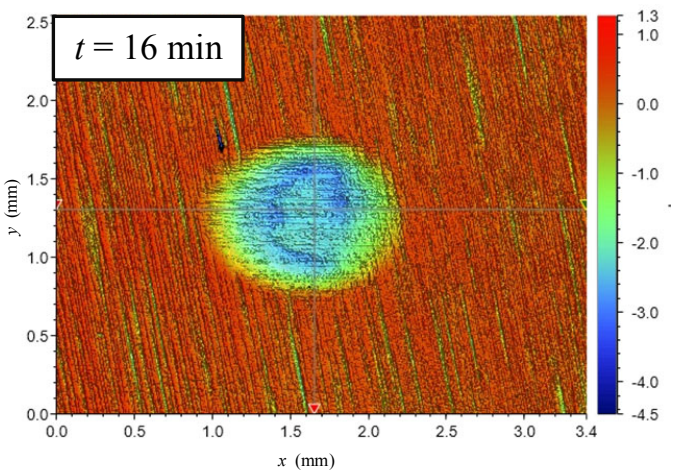

those of the middle area, and the wear section is approximately axisymmetric. This is because under mixed lubrication, the contact area is elastically deformed, and a horseshoe-shaped oil vortex is formed under EHL pressure, causing a higher $h$ than that of the edge region (a more detailed analysis is shown in Ref. [18]). Although the distribution of asperity is random, the pressure and temperature distributions are approximately bilaterally symmetric. The predicted wear track shows good agreement with the experiments. Although the profiles predicted by the Arrhenius relationship are slightly deeper, the difference is not significant.

Figure 7 shows the evolution of the $h, p$, and flash temperature $\left(T_{\mathrm{f}}\right)$ during the wear evolution. In the central area, $h$ is low before the introduction of wear, as a result of the asperity contact. After the run-in period, the lubrication state in the central area is improved. The asperity areas become smaller and the pressure distribution becomes smoother. During wear evolution, the contact area gradually expands, and more asperity contact occurs in the outer ring of the contact region. The wear is dominated by the contact pressure because the flash temperature in the contact region is relatively low. 


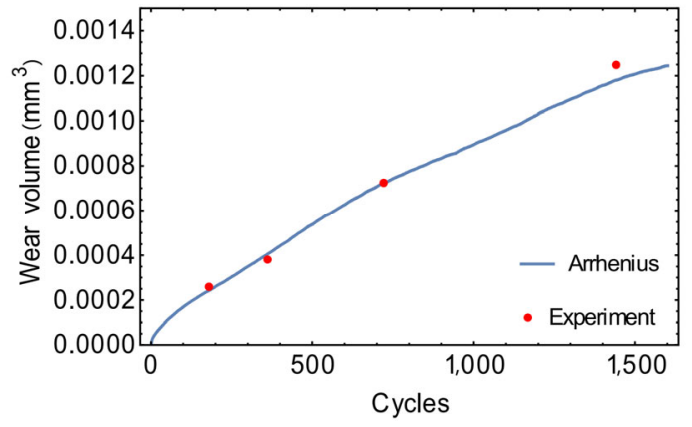

Fig. 5 Wear volume comparison.

Figure 8 shows a two-dimensional (2D) view of $h$ and $p$ evolutions at the center section in the speed direction. $X$ is the dimensionless length in $x$ direction. The changes in $h$ and $p$ are clearly shown.

According to the above study, the model can accurately predict the surface topography evolution over time. To further investigate the performance of the wear model under different speed and load, the following comparisons are conducted using the experiment results. Furthermore, the simulations are conducted at different temperatures, and a

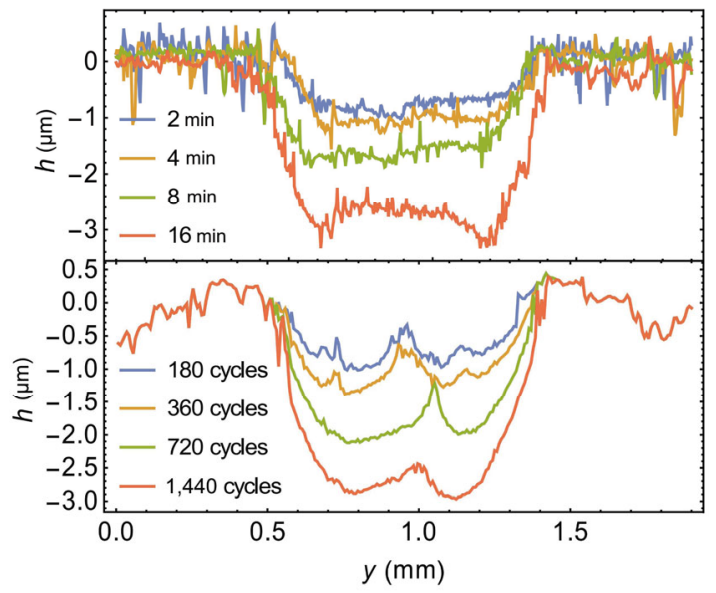

Fig. 6 Center profiles of the experiment and Arrheniusbased model.
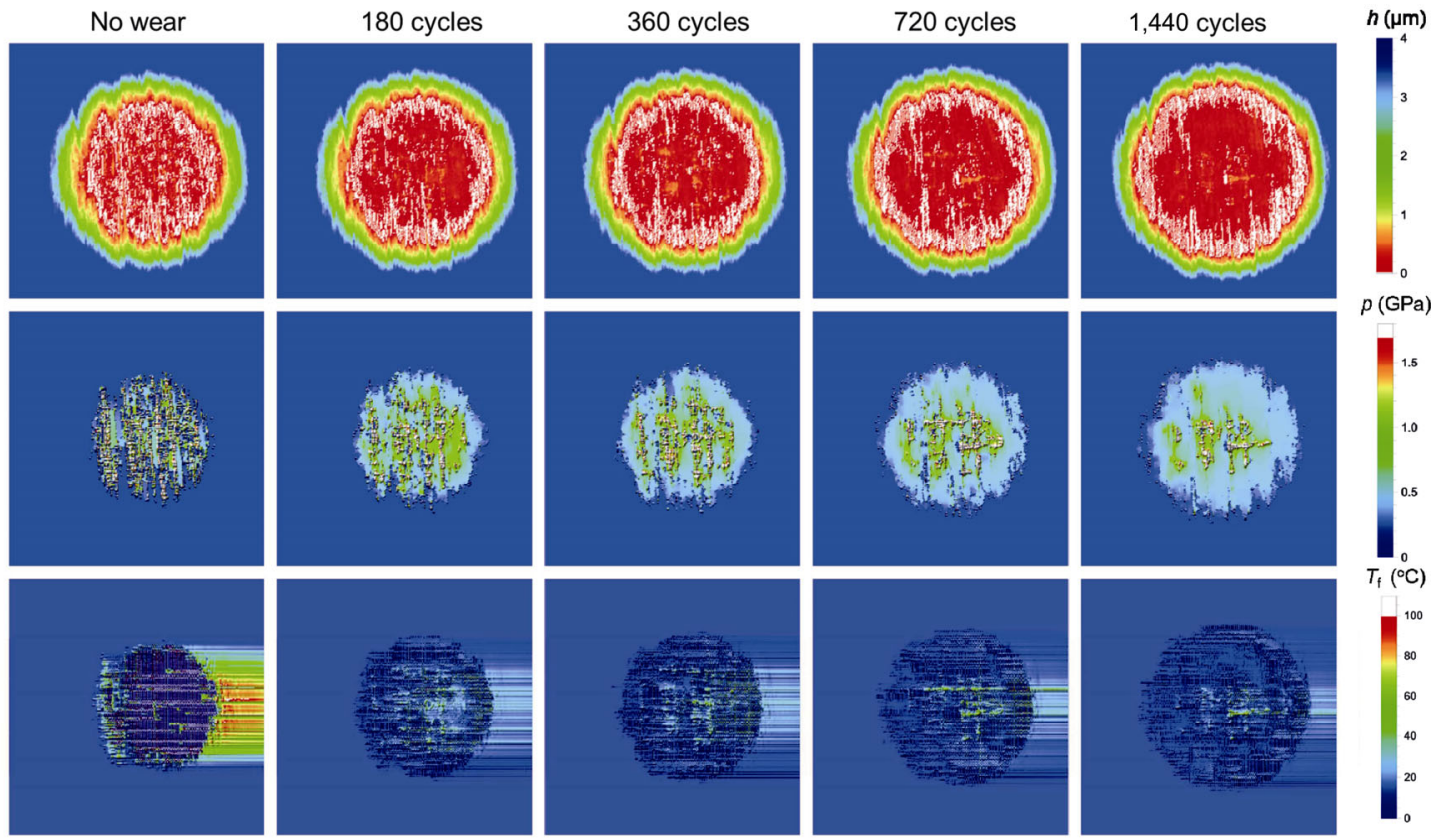

Fig. 7 Evolutions of $h, p$, and $T_{\mathrm{f}}$ over time.

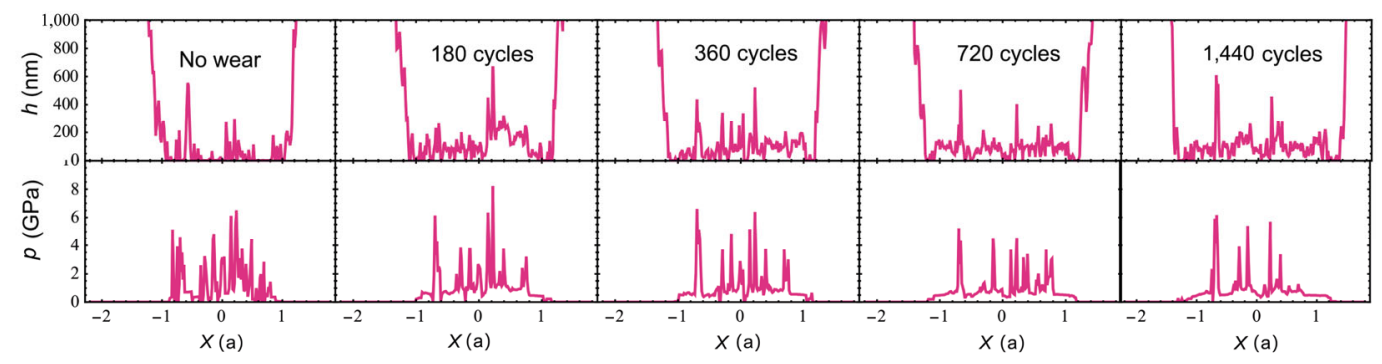

Fig. 8 2D view of $h$ (top) and $p$ evolutions (bottom). 
qualitative comparison of the available test data in the literature is performed.

\subsection{Comparison of wear profiles under diffe- rent speeds}

This section focuses on a comparison of the effect of speed on the wear profiles after sliding the same distance. The disk is fixed, the load is $500 \mathrm{~N}$, and the velocities of the ball are $0.05,0.1,0.2$, and 0.4 $\mathrm{m} / \mathrm{s}$ in the experiments. The total running distance is $96 \mathrm{~m}$, corresponding to the running time of 32, 16, 8 , and $4 \mathrm{~min}$ for the four different velocities. The acceleration factors used in the simulation are the same as those in Section 4.1, and the number of wear simulation cycles is 720 .

Figure 9 shows the wear topography at four different speeds with the same sliding distance and load. The speed has a significant effect on the wear behavior under mixed lubrication. The wear decreases with increasing speed. When the speed is higher than $0.1 \mathrm{~m} / \mathrm{s}$, the central part has the same convexity as that in Section 4.1. However, when the speed is $0.05 \mathrm{~m} / \mathrm{s}$, no bump is observed in the central part. The bump in the central part is caused by the uneven distribution of the asperity contact region.

Figure 10 shows the wear profiles of the experiment and the wear profiles predicted by the Arrhenius relationship. The wear depth at different speeds is consistent. However, the wear tracks simulated at low speeds are deeper than those of the experiments, suggesting that the acceleration factor may need to be re-determined at different entrainment speeds for greater precision. The difference of prediction effects under different speeds is most likely caused by three-body wear, which is ignored in our model. At low speeds, the flow of oil is slow, and the wear debris is difficult to discharge. Typically, the wear debris hinders the formation of the lubrication film and weakens the lubrication effect. However, the wear debris can also protect the surfaces and reduce wear.

Figure 11 shows a comparison of the wear volume at different velocities after the same relative sliding distances. The model can accurately fit the experiment in the speed range of $0.1-0.4 \mathrm{~m} / \mathrm{s}$ using the acceleration factor measured under $0.2 \mathrm{~m} / \mathrm{s}$. When the speed is reduced to $0.05 \mathrm{~m} / \mathrm{s}$, the predicted wear volume is higher than that of the experiment. Overall, the wear volume prediction is effective.
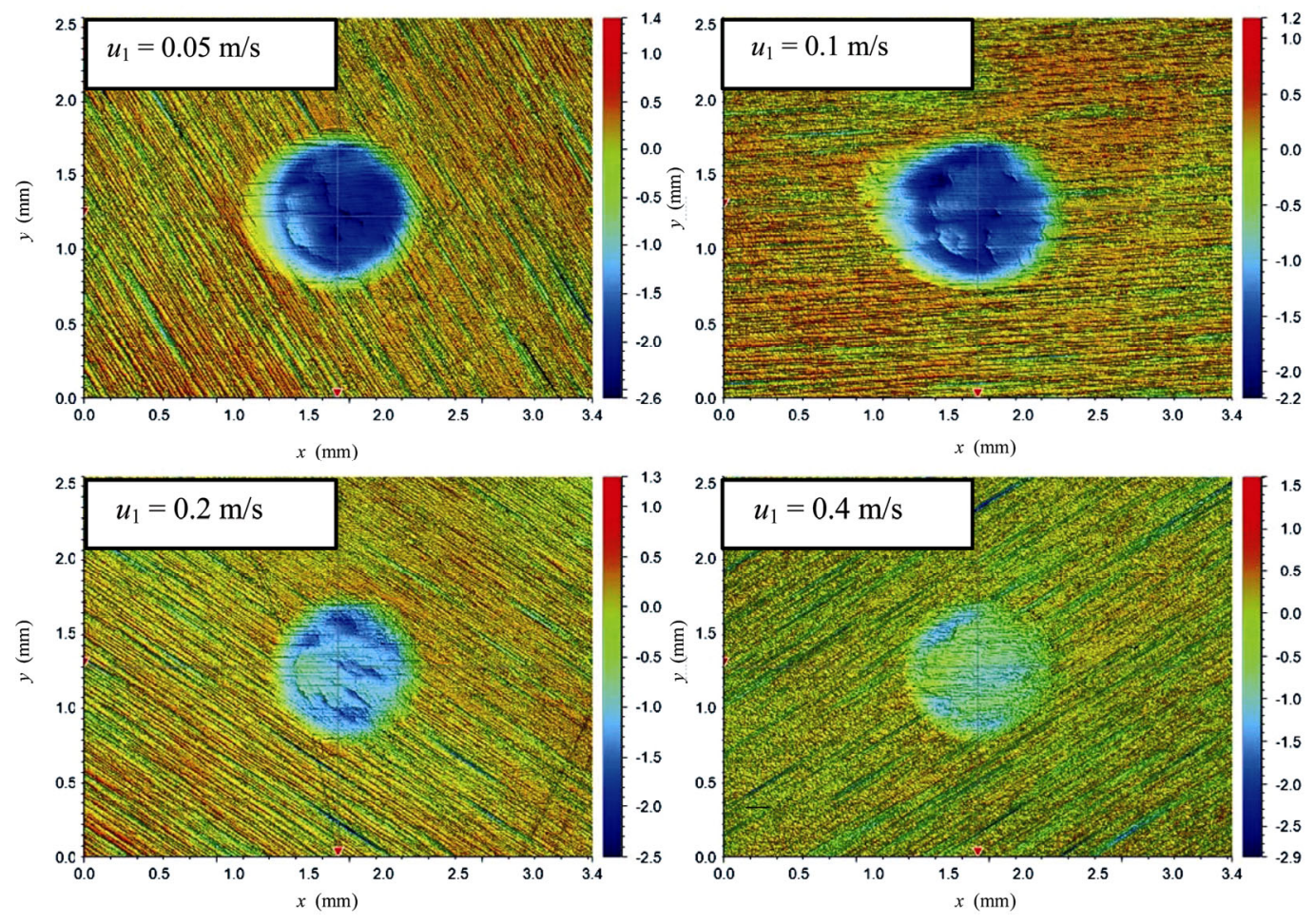

Fig. 9 Disk surfaces after wear at different speeds. 


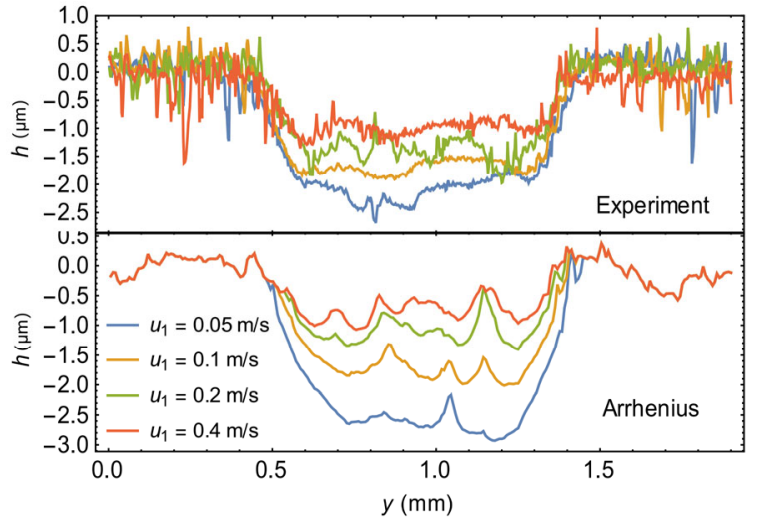

Fig. 10 Comparison of the wear profiles on the center section perpendicular to the speed.

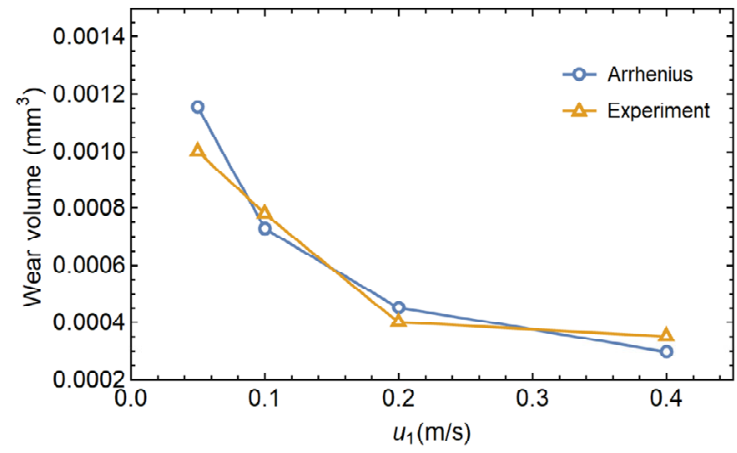

Fig. 11 Wear volume vs. speed.

\subsection{Comparison of wear profiles under diffe- rent loads}

A comparison of the wear profile under different loads after sliding the same distance is shown. The disk is fixed. The speed of the ball is $0.2 \mathrm{~m} / \mathrm{s}$, and the loads of ball are 200,400, and $600 \mathrm{~N}$. The total running time is $10 \mathrm{~min}$. The acceleration factor used in the simulation is the same as that in Section 4.1, and the number of wear simulation cycles is 900 .

The load affects wear under mixed lubrication. As shown in Fig. 12, the width of the wear profiles increases when the load increases from 200 to $600 \mathrm{~N}$.

Figure 13 shows the wear profiles of the experiment and the wear profiles predicted by the Arrhenius relationship. Although the profile sizes under the three different loads are different, the measured wear depths in the center are similar. This may be because the load influence on the oil film thickness is not significant. Although the load affects contact fatigue, the fatigue wear testing in $10 \mathrm{~min}$ may be limited. From Fig. 13, the general characteristics of the wear profile under different loads are consistent with the experimental values, except for some local details after $10 \mathrm{~min}$ of wear.

Figure 14 shows a comparison of the wear volume at different loads after the same sliding distances. The model can fit the experiments under different loads using the acceleration factor measured under the load. The wear volume is linear with the load. The wear volume predicted at a high load is more accurate than that at a low load.

\subsection{Comparison of wear volumes at different temperatures}

To maintain wear formation, the rubbing surfaces should work in mixed or boundary lubrication, indicating low entraining and sliding speeds. As under the room-temperature condition, a high asperity contact temperature is difficult to obtain with a low sliding speed. Limited by the experimental conditions, the wear at different working temperatures $(T)$ is studied through simulation only. The simulation parameters are the same as those in Section 4.1. The simulation temperature of the ball and disk ranges from 40 to $70{ }^{\circ} \mathrm{C}$. The number of simulation cycles is 400 .
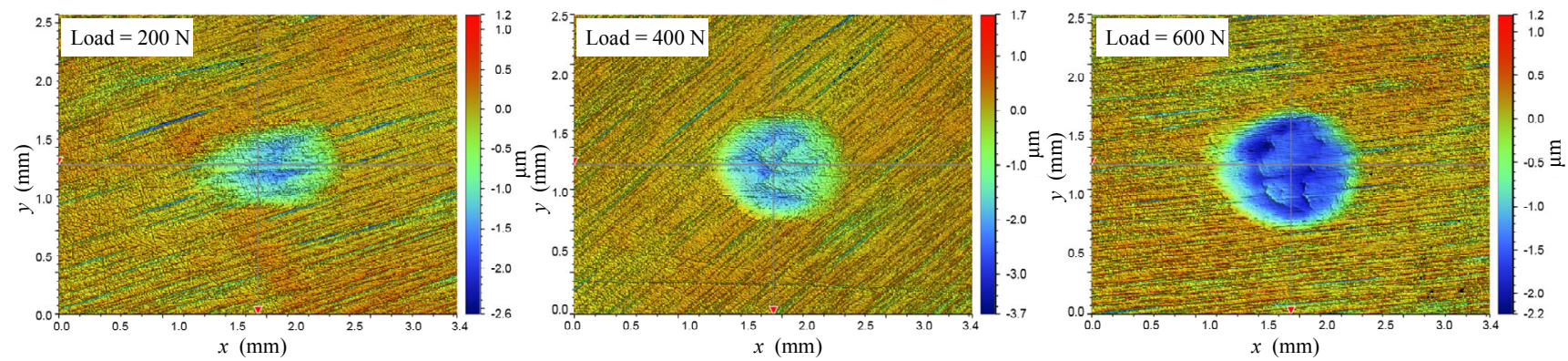

Fig. 12 Disk surfaces after wear at different loads. 


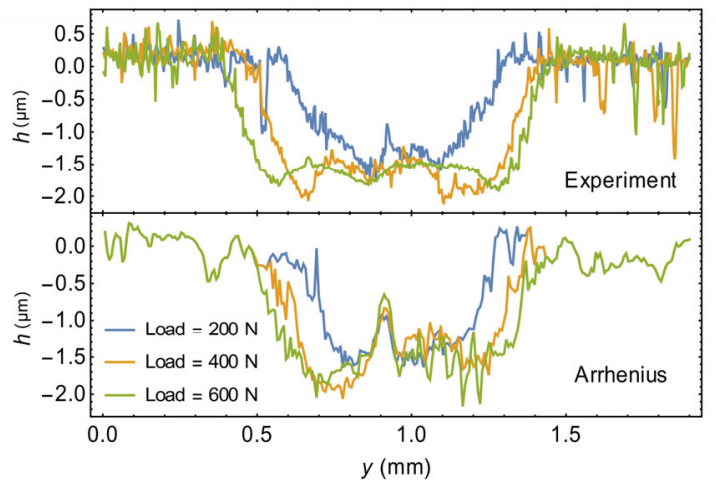

Fig. 13 Comparison of the wear profile on the center section perpendicular to speed.

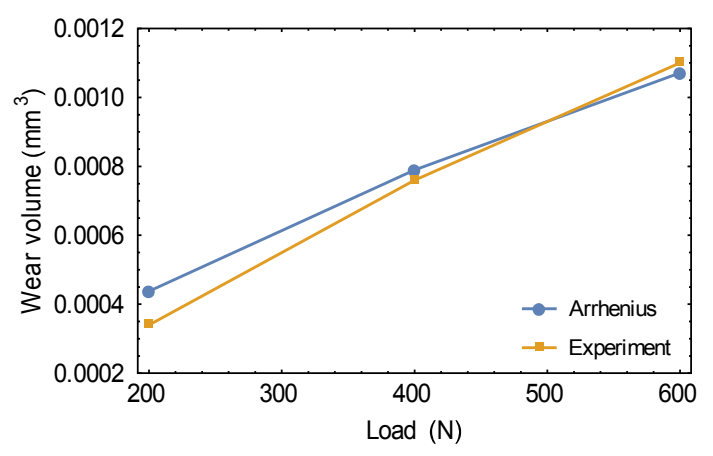

Fig. 14 Wear volume vs. load.

Figure 15 suggests that the wear volume has an approximately linear relationship with the temperature. Besides, the wear rate shows an exponential relationship with the temperature because the high temperature
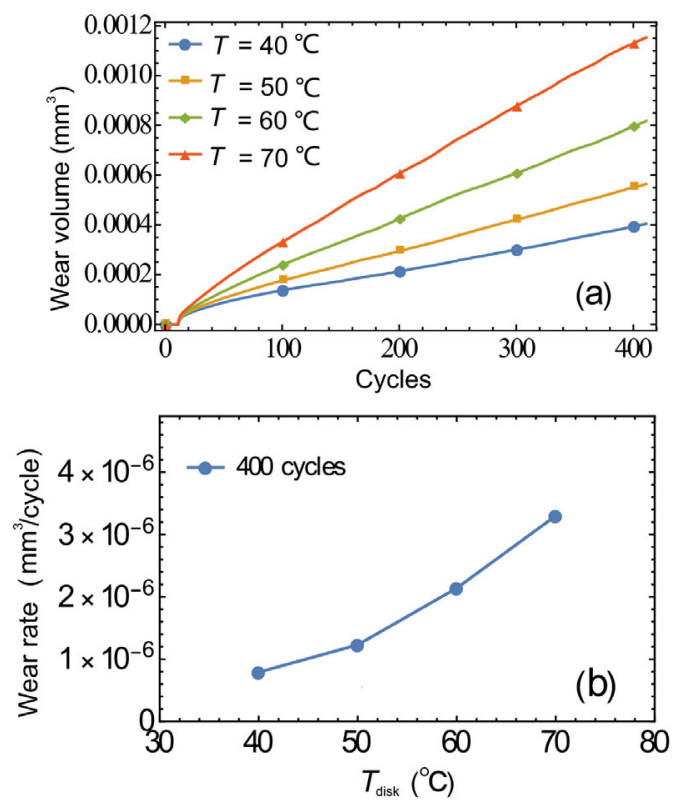

Fig. 15 (a) Wear volumes vs. cycles and (b) wear rate vs. temperature. can accelerate the wear reaction rate, as shown in Eq. (7). The wear rate law has been verified in Ref. [24]. Lancaster [31] reported similar findings when studying the dry contact wear of metals through experiments. He found that when the temperature is less than $300{ }^{\circ} \mathrm{C}$, the wear rate increases exponentially by increasing the temperature, as shown in Fig. 16.

\subsection{Comparison of the mixed EHL wear model with Archard's}

The mixed EHL wear model is also compared with Zhu's Archard-based mixed lubrication wear model [17]. The simulation and experimental conditions are the same as those in Section 4.1. To fit the experimental curve, the acceleration factor for the disk is 0.0625 , based on the Archard equation.

As shown in Fig. 17, the wear volume predicted by the Arrhenius relationship is closer to the experimental results than that predicted by the Archard theory. However, the difference is not large. The curve predicted by the Archard theory is also similar to that of the experimental results. The wear rate based on the Archard relationship is slightly higher initially. The wear rates of both models decrease as wear progresses; however, the

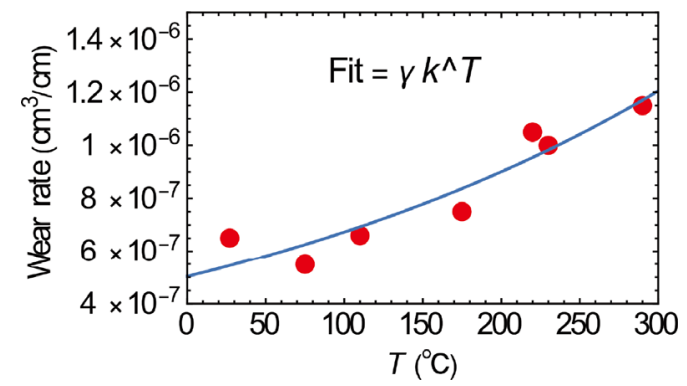

Fig. 16 Wear rate with temperature for $60 / 40$ brass on tool steel. Reproduced with permission from Ref. [31], C) Springer Nature 2020.

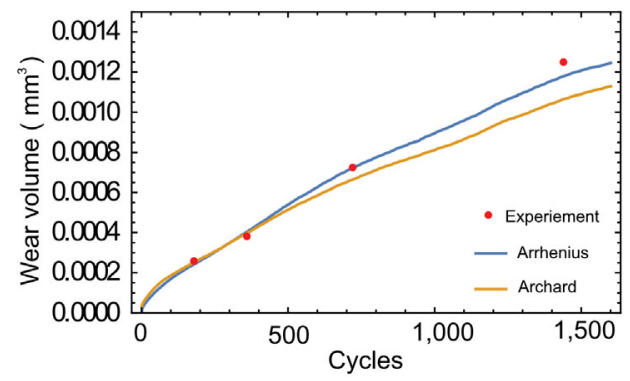

Fig. 17 Wear volume evolution comparison of the experiment and the two models (Archard and Arrhenius). 


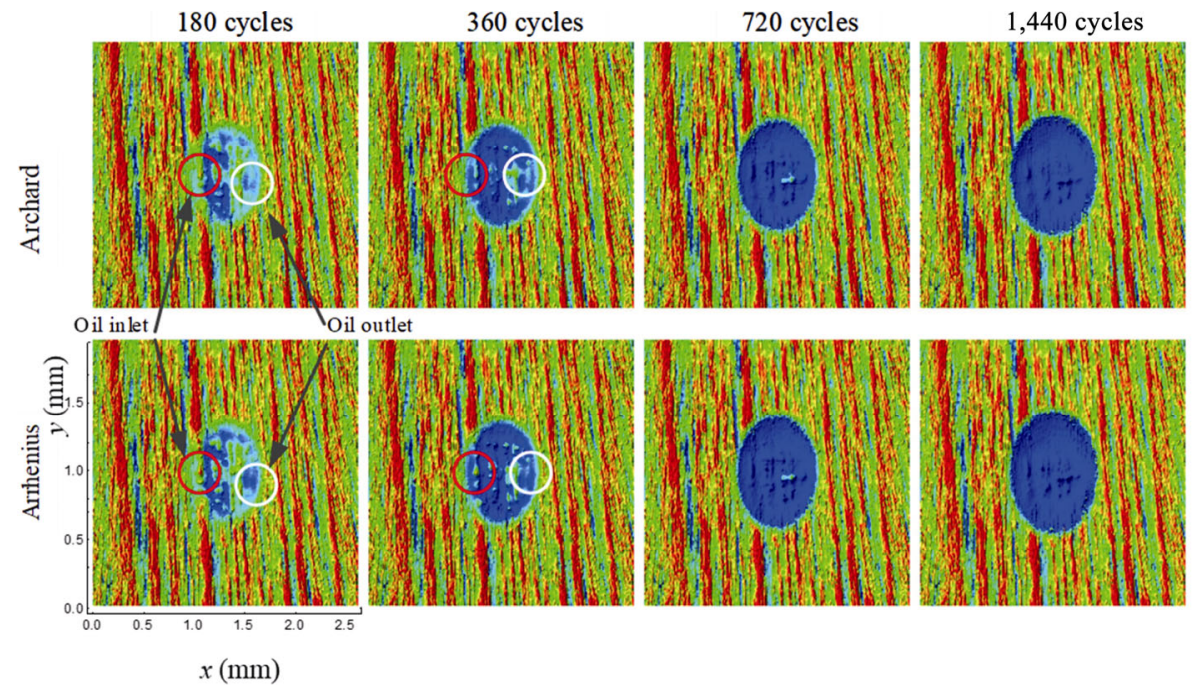

Fig. 18 Simulation of disk surfaces.

Archard rate decreases faster. Thus, the model based on the Archard theory may underestimate wear. This may be because the Archard model primarily considers adhesive wear by force; however, more types of wear occur in the experiment.

The predicted surface topographies of the two models are shown in Fig. 18. The predicted profiles of the two models are approximately the same; however, there are some differences. The wear at the entrance (marked by a red circle) predicted by Archard is higher than that of Arrhenius. By contrast, the opposite correlation is observed at the exit (marked by a white circle). This phenomenon is initially apparent; however, as wear progresses, the difference diminishes. The pressure rapidly decreases to zero along the exit direction; however, the flash temperature does not. This may be the largest difference between the two models. However, because the early wear is shallow and the random fluctuations are large, the current experiment cannot determine the more realistic model. Conversely, as demonstrated in Ref. [26], the variation of wear rate vs. temperature is an exponential relationship. Temperature is not considered in the Archard theory, indicating that with high asperity contact temperature, the wear profile prediction by the energy-based Arrhenius equation is more reasonable than that by the Archard wear equation.

\section{Conclusions}

A new wear prediction approach based on the energy relationship first proposed by Arrhenius has been evaluated in this study. Through experiments and simulations, the effects of time, load, and speed on the wear behavior of the disk have been studied. Comparisons of the experiments and simulations have shown that the model is effective. The following conclusions can be drawn:

1) The mixed EHL wear model based on the Arrhenius relationship is effective, and it can accurately predict wear evolution.

2) Velocity has a strong influence on wear depth, while the load can affect the width of the wear track. However, the load's effect on the sliding wear depth is not significant in mixed lubrication.

3) The acceleration coefficient measured under certain working conditions also agrees well with the experimental results under different loads; however, the predictions may not be accurate when the same acceleration coefficient is applied at low speeds, which may be caused by abrasive wear.

4) The predictions based on Arrhenius and Archard are all credible. The Arrhenius relationship is more accurate in wear volume prediction.

\section{Acknowledgements}

The authors acknowledge the support from the National Natural Science Foundation of China (NSFC) (51875369) and the Central Universities Funds (YJ201752). Wei PU would like to express his appreciation for Prof. Dong ZHU's advice in 
the mixed EHL model.

Open Access This article is licensed under a Creative Commons Attribution 4.0 International License, which permits use, sharing, adaptation, distribution and reproduction in any medium or format, as long as you give appropriate credit to the original author(s) and the source, provide a link to the Creative Commons licence, and indicate if changes were made.

The images or other third party material in this article are included in the article's Creative Commons licence, unless indicated otherwise in a credit line to the material. If material is not included in the article's Creative Commons licence and your intended use is not permitted by statutory regulation or exceeds the permitted use, you will need to obtain permission directly from the copyright holder.

To view a copy of this licence, visit http://creativecommons.org/licenses/by/4.0/.

\section{References}

[1] Meng H C, Ludema K C. Wear models and predictive equations: Their form and content. Wear 181-183: 443457 (1995)

[2] Archard J F. Contact and rubbing of flat surfaces. J Appl Phys 24(8): 981-988 (1953)

[3] Andersson J, Almqvist A, Larsson R. Numerical simulation of a wear experiment. Wear 271(11-12): 2947-2952 (2011)

[4] Binnig G, Quate C F, Gerber C. Atomic force microscope. Phys Rev Lett 56(9): 930-933 (1986)

[5] Gotsmann B, Lantz M A. Atomistic wear in a single asperity sliding contact. Phys Rev Lett 101(12): 125501 (2008)

[6] Laidler K J. The development of the Arrhenius equation. J Chem Educ 61(6): 494 (1984)

[7] Jacobs T D B, Carpick R W. Nanoscale wear as a stress-assisted chemical reaction. Nat Nanotechnol 8(2): 108-112 (2013)

[8] Liu J, Grierson D S, Moldovan N, Notbohm J, Li S, Jaroenapibal P, O'Connor S D, Sumant A V, Neelakantan $\mathrm{N}$, Carlisle J A, et al. Preventing nanoscale wear of atomic force microscopy tips through the use of monolithic ultrananocrystalline diamond probes. Small 6(10): 11401149 (2010)

[9] Bhaskaran H, Gotsmann B, Sebastian A, Drechsler U, Lantz M A, Despont M, Jaroenapibal P, Carpick R W, Chen Y, Sridharan K. Ultralow nanoscale wear through atom-by-atom attrition in silicon-containing diamond-like carbon. Nat Nanotechnol 5(3): 181-185 (2010)

[10] Valentin L P, Roman P. Adhesive wear and particle emission: Numerical approach based on asperity-free formulation of Rabinowicz criterion. Friction 6(3): 260273 (2018)

[11] Jean-François M, Ramin A, Tobias B, Lucas F, Enrico M. Adhesive wear mechanisms uncovered by atomistic simulations. Friction 6(3): 245-259 (2018)

[12] Souilliart T, Rigaud E, Le Bot A, Phalippou C. Energybased wear law for oblique impacts in dry environment. Tribol Int 105: 241-249 (2017)

[13] Ghanbarzadeh A, Wilson M, Morina A, Dowson D, Neville A. Development of a new mechano-chemical model in boundary lubrication. Tribol Int 93: 573-582 (2016)

[14] Hu Y Z, Zhu D. A full numerical solution to the mixed lubrication in point contacts. J Tribol 122(1): 1-9 (2000)

[15] Hooke C J, Li K G, Morales-Espejel G. Rapid calculation of the pressures and clearances in rough, rolling-sliding elastohydrodynamically lubricated contacts. Part 2: General, non-sinusoidal roughness. Proc Inst Mech Eng Part C J Mech Eng Sci 221(5): 551-562 (2007)

[16] Masjedi M, Khonsari M M. On the effect of surface roughness in point-contact EHL: Formulas for film thickness and asperity load. Tribol Int 82: 228-244 (2015)

[17] Zhu D, Martini A, Wang W Z, Hu Y Z, Lisowsky B, Wang Q J. Simulation of sliding wear in mixed lubrication. J Tribol 129(3): 544-552 (2007)

[18] Pei X, Pu W, Zhang Y, Huang L. Surface topography and friction coefficient evolution during sliding wear in a mixed lubricated rolling-sliding contact. Tribol Int 137: 303-312 (2019)

[19] Zhao E H, Ma B, Li H Y. Wear and lubrication behaviors of $\mathrm{Cu}$-based friction pairs with asperity contacts: Numerical and experimental studies. Tribol Lett 65(2): 69 (2017)

[20] Morales-Espejel G E, Rycerz P, Kadiric A. Prediction of micropitting damage in gear teeth contacts considering the concurrent effects of surface fatigue and mild wear. Wear 398-399: 99-115 (2018)

[21] Lewis R. A modelling technique for predicting compound impact wear. Wear 262(11-12): 1516-1521 (2007)

[22] Bazrafshan M, De Rooij M B, Valefi M, Schipper D J. Numerical method for the adhesive normal contact analysis based on a Dugdale approximation. Tribol Int 112: 117-128 (2017)

[23] Maugis D. Adhesion of spheres: The JKR-DMT transition using a Dugdale model. J Colloid Interface Sci 150(1): 243-269 (1992)

[24] Akchurin A, Bosman R. A deterministic stress-activated model for tribo-film growth and wear simulation. Tribol Lett 65(2): 59 (2017) 
[25] Furustig J, Almqvist A, Bates C A, Ennemark P, Larsson R. A two scale mixed lubrication wearing-in model, applied to hydraulic motors. Tribol Int 90: 248-256 (2015)

[26] Chung K H. Wear characteristics of atomic force microscopy tips: A review. Int J Precis Eng Manuf 15(10): 22192230 (2014)

[27] Bair S, Winer W O. A rheological model forelastohydrodynamic contacts based on primary laboratory data. ASME J Lubr Technol 101: 258-264 (1979)

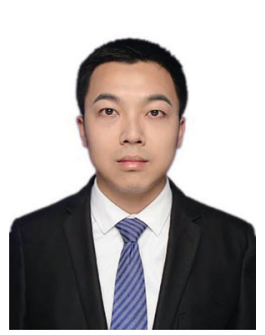

Xin PEI. He is a Ph.D. candidate studying at Sichuan University, China. His research interests include friction, lubrication, wear, and dynamics. Since he joined the group of Prof. Wei PU in 2018, he has been conducting experimental

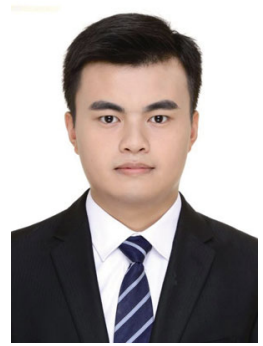

Wei PU. He is a full professor at Sichuan University, China, a postdoctoral fellow at Massachusetts Institute of Technology, the chapter president of the $6^{\text {th }}$ World Tribology Congress. He has won the Tsinghua University's Wen
[28] Dyson A. Frictional traction and lubricant rheology in elastohydrodynamic lubrication. Phil Trans Roy Soc Lond A 266(1170): 1-33 (1970)

[29] Carslaw H S, Jaeger J C. Conduction of Heat in Solids. 2nd ed. Oxford (Britain): Clarendon Press, 1959.

[30] Liu Y C, Wang H, Wang W Z, Hu Y Z, Zhu D. Methods comparison in computation of temperature rise on frictional interfaces. Tribol Int 35(8): 549-560 (2002)

[31] Lancaster J K. The influence of temperature on metallic wear. Proc Phys Soc Sect B 70(1): 112-118 (1957)

and numerical research on a variety of tribology related industry problems.

Shi Zhu Maple Leaf Award- Outstanding Young Scholar Award. He is mainly engaged in scientific research of transmission, tribology, molecular dynamics, and nanomaterials. He has published more than $30 \mathrm{SCI} / \mathrm{EI}$ papers as the first/corresponding author. 